\title{
On the average number of unitary factors of finite abelian groups
}

by

\author{
J. Wu (Nancy)
}

1. Introduction. Let $\mathbb{X}$ be the semigroup of all finite abelian groups with respect to the direct product $\otimes$ and let $\mathcal{E}_{0}$ be the identity of $\mathbb{X}$. For $\mathcal{G} \in \mathbb{X}$ and $\mathcal{H} \in \mathbb{X}$, we use $(\mathcal{G}, \mathcal{H})$ to denote the group of maximal order in $\mathbb{X}$ which is simultaneously a direct factor of $\mathcal{G}$ and $\mathcal{H}$. We say that $\mathcal{G}$ and $\mathcal{H}$ are relatively prime if $(\mathcal{G}, \mathcal{H})=\mathcal{E}_{0}$. A direct factor $\mathcal{D}$ of $\mathcal{G}$ is called unitary if $\mathcal{D} \otimes \mathcal{E}=\mathcal{G}$ and $(\mathcal{D}, \mathcal{E})=\mathcal{E}_{0}$. The number of unitary factors of $\mathcal{G}$ is denoted by $t(\mathcal{G})$. In 1960 , Cohen [2] proved

$$
\sum_{|\mathcal{G}| \leq x} t(\mathcal{G})=A_{1} x \log x+A_{2} x+O(\sqrt{x} \log x),
$$

where the summation is over all $\mathcal{G}$ in $\mathbb{X}$ of order $|\mathcal{G}| \leq x$ and the $A_{j}$ are some effective constants. After a study on Dirichlet's series associated with $t(\mathcal{G})$, Krätzel [7] found a connection between (1.1) and the following threedimensional divisor problem:

$$
\sum_{n_{1} n_{2} n_{3}^{2} \leq x} 1=B_{1} x \log x+B_{2} x+B_{3} \sqrt{x}+\Delta(1,1,2 ; x),
$$

where the $B_{j}$ are some effective constants and $\Delta(1,1,2 ; x)$ is an error term. Using exponential sum techniques, he showed that $\Delta(1,1,2 ; x) \ll$ $x^{11 / 29}(\log x)^{2}$, which implies

$$
\sum_{|\mathcal{G}| \leq x} t(\mathcal{G})=A_{1} x \log x+A_{2} x+A_{3} \sqrt{x}+\Delta(x)
$$

with $\Delta(x) \ll x^{11 / 29}(\log x)^{2}$. This estimate was improved to $\Delta(x) \ll$ $x^{3 / 8}(\log x)^{4}$ by Schmidt [11], then to $\Delta(x) \ll_{\varepsilon} x^{77 / 208+\varepsilon}$ by Liu [9] and to $\Delta(x) \ll_{\varepsilon} x^{29 / 80+\varepsilon}$ by Liu [10], where $\varepsilon$ denotes an arbitrarily small positive number.

1991 Mathematics Subject Classification: 11L07, 11N45. 
In this paper, we give a better bound.

TheOrem 1. For any $\varepsilon>0$, we have

$$
\Delta(1,1,2 ; x) \ll_{\varepsilon} x^{47 / 131+\varepsilon} \quad \text { and } \Delta(x) \ll_{\varepsilon} x^{47 / 131+\varepsilon} .
$$

For comparison, we have $29 / 80=0.3625$ and $47 / 131 \approx 0.3587$. From [10], we know that in the proof of Theorem 1 the most difficult part is to estimate the exponential sums of type

$$
\sum_{h \sim H} a_{h} \sum_{n_{1} \sim N_{1}} \sum_{n_{2} \sim N_{2}} e\left(x h /\left(n_{1}^{2} n_{2}\right)\right),
$$

where $\left|a_{h}\right| \leq 1, e(t):=e^{2 \pi i t}$ and the notation $h \sim H$ means $c H<h \leq c^{\prime} H$ with some positive unspecified constants $c, c^{\prime}$. Liu [10] has treated (1.4) by combining Fouvry-Iwaniec's method [3] and Kolesnik's method [6].

We notice that via van der Corput's $B$-process the sum (1.4) can be transformed into bilinear exponential sums of type I,

$$
T(M, N):=\sum_{m \sim M} \sum_{n \in I(m)} \varphi_{m} e\left(X \frac{m^{\alpha} n^{\beta}}{M^{\alpha} N^{\beta}}\right),
$$

where $I(m)$ is a subinterval of $[N, 2 N]$. Using the classical $A, B$ process and the well known AB theorem of Kolesnik (see Theorem 1 of $[6]$ and Lemma 1.5 of [8]) we shall prove an estimate for $T(M, N)$ (see Theorem 3 below). In addition we also use an idea of Jia ([5], Lemma 13) and Liu ([8], Lemma 2.4) to investigate bilinear exponential sums of type II,

$$
S(M, N):=\sum_{m \sim M} \sum_{n \sim N} \varphi_{m} \psi_{n} e\left(X \frac{m^{\alpha} n^{\beta}}{M^{\alpha} N^{\beta}}\right) .
$$

Baker and Harman have simplified Jia-Liu's argument to obtain a slightly more general estimate for $S(M, N)$ (see Theorem 2 of [1]) than those of Jia and Liu. But all such results contain some restrictions on $(X, M, N)$ and the number of terms is relatively large; this is not convenient in applications. Our result (see Theorem 2 below) essentially has the same power as their estimates, but it is without restriction, more general and simpler in form. Finally, it is worth indicating that we also need Theorem 7 of [12] and Lemma 2.3 of [13] for the proof of Theorem 1.

2. Estimates for exponential sums. We first prove two estimates for $S=S(M, N)$, defined as in Section 1. In the sequel, the letter $\varepsilon_{0}$ denotes a suitably small positive number (depending on $\alpha, \beta$ and $\alpha_{j}$ at most).

Theorem 2. Let $\alpha, \beta \in \mathbb{R}$ with $\alpha \beta(\alpha-1)(\beta-1) \neq 0, X>0, M \geq 1$, $N \geq 1,\left|\varphi_{m}\right| \leq 1,\left|\psi_{n}\right| \leq 1$ and $\mathcal{L}:=\log (2+X M N)$. If $(\kappa, \lambda)$ is an exponent pair, then 


$$
\begin{aligned}
S(M, N) \ll & \left\{\left(X^{2+4 \kappa} M^{8+10 \kappa} N^{9+11 \kappa+\lambda}\right)^{1 /(12+16 \kappa)}\right. \\
& +X^{1 / 6} M^{2 / 3} N^{3 / 4+\lambda /(12+12 \kappa)} \\
& +\left(X M^{3} N^{4}\right)^{1 / 5}+\left(X M^{7} N^{10}\right)^{1 / 11} \\
& +M^{2 / 3} N^{11 / 12+\lambda /(12+12 \kappa)} \\
& \left.+M N^{1 / 2}+\left(X^{-1} M^{14} N^{23}\right)^{1 / 22}+X^{-1 / 2} M N\right\} \mathcal{L}^{2}, \\
S(M, N) \ll & \left\{\left(X M^{3} N^{4}\right)^{1 / 5}+\left(X^{4} M^{10} N^{11}\right)^{1 / 16}+\left(X M^{7} N^{10}\right)^{1 / 11}\right. \\
& \left.+M N^{1 / 2}+\left(X^{-1} M^{14} N^{23}\right)^{1 / 22}+X^{-1 / 2} M N\right\} \mathcal{L}^{2} .
\end{aligned}
$$

Proof. We begin in the same way as Jia [5], Liu [8], Baker and Harman [1]. Without loss of generality, we suppose that $\beta>0$ and $\mathcal{L}$ is sufficiently large. Let $Q \in[\mathcal{L}, N / \mathcal{L}]$ be a parameter to be chosen later. By Cauchy's inequality and a "Weyl shift" ([4], Lemma 2.5), we have

$$
\begin{aligned}
|S|^{2} \ll & \frac{(M N)^{2}}{Q} \\
& +\frac{M^{3 / 2} N}{Q} \sum_{1 \leq\left|q_{1}\right|<Q}\left(1-\frac{\left|q_{1}\right|}{Q}\right) \sum_{n+q_{1}, n \sim N} \psi_{n+q_{1}} \bar{\psi}_{n} \sum_{m \sim M} m^{-1 / 2} e\left(A m^{\alpha} t\right),
\end{aligned}
$$

where $t=t\left(n, q_{1}\right):=\left(n+q_{1}\right)^{\beta}-n^{\beta}$ and $A:=X /\left(M^{\alpha} N^{\beta}\right)$. Splitting the range of $q_{1}$ into dyadic intervals and removing $1-q_{1} / Q$ by partial summation, we get

$$
|S|^{2} \ll(M N)^{2} Q^{-1}+\mathcal{L} M^{3 / 2} N Q^{-1} \max _{1 \leq Q_{1} \leq Q}\left|S\left(Q_{1}\right)\right|
$$

where

$$
S\left(Q_{1}\right):=\sum_{q_{1} \sim Q_{1}} \sum_{n+q_{1}, n \sim N} \psi_{n+q_{1}} \bar{\psi}_{n} \sum_{m \sim M} m^{-1 / 2} e\left(A m^{\alpha} t\right)
$$

If $X(M N)^{-1} Q_{1} \geq \varepsilon_{0}$, by Lemma 2.2 of [12] we can transform the innermost sum to a sum over $l$ and then using Lemma 2.3 of [12] with $n=m$ we can estimate the corresponding error term. As a result, we obtain

$$
\begin{aligned}
S\left(Q_{1}\right) \ll & \sum_{q_{1} \sim Q_{1}} \sum_{n+q_{1}, n \sim N} \psi_{n+q_{1}} \bar{\psi}_{n} \sum_{l \in I} l^{-1 / 2} e\left(\widetilde{\alpha}(A t)^{\gamma} l^{1-\gamma}\right) \\
& +\left\{\left(X M^{-1} N^{-1} Q_{1}^{3}\right)^{1 / 2}+M^{-1 / 2} N Q_{1}\right. \\
& \left.+\left(X^{-1} M N Q_{1}\right)^{1 / 2}+\left(X^{-2} M N^{4}\right)^{1 / 2}\right\} \mathcal{L},
\end{aligned}
$$

where $\gamma:=1 /(1-\alpha), \widetilde{\alpha}=|1-\alpha| \cdot|\alpha|^{\alpha /(1-\alpha)}, I:=\left[c_{1} A M^{\alpha-1}|t|, c_{2} A M^{\alpha-1}|t|\right]$ and $c_{j}=c_{j}(\alpha)$ are some constants. Exchanging the order of summation and estimating the sum over $l$ trivially, we find, for some $l \asymp X(M N)^{-1} Q_{1}$, the 
inequality

$$
\begin{aligned}
S\left(Q_{1}\right) \ll & \left(X M^{-1} N^{-1} Q_{1}\right)^{1 / 2}\left|\sum_{\left(n, q_{1}\right) \in \mathbf{D}_{1}(l)} \psi_{n+q_{1}} \bar{\psi}_{n} e\left(\widetilde{\alpha}(A t)^{\gamma} l^{1-\gamma}\right)\right| \\
& +\left\{\left(X M^{-1} N^{-1} Q_{1}^{3}\right)^{1 / 2}+M^{-1 / 2} N Q_{1}\right. \\
& \left.+\left(X^{-1} M N Q_{1}\right)^{1 / 2}+\left(X^{-2} M N^{4}\right)^{1 / 2}\right\} \mathcal{L},
\end{aligned}
$$

where $\mathbf{D}_{1}(l)$ is a suitable subregion of $\left\{\left(n, q_{1}\right): n \sim N, q_{1} \sim Q_{1}\right\}$. Let $S_{1}\left(Q_{1}\right)$ be the double sums on the right-hand side of (2.4). Using Lemma 2.6 of [12] to relax the range of $q_{1}$, we see that there exists a real number $\theta$ independent of $\left(n, q_{1}\right)$ such that

$$
S_{1}\left(Q_{1}\right) \ll \mathcal{L} \sum_{n \sim N}\left|\sum_{q_{1} \sim Q_{1}} \psi_{n+q_{1}} e\left(\theta q_{1}\right) e\left(\widetilde{\alpha}(A t)^{\gamma} l^{1-\gamma}\right)\right| .
$$

If $\mathcal{L} \leq Q_{1} \leq Q$, using again Cauchy's inequality and a "Weyl shift" with $Q_{2} \leq \varepsilon_{0} \sqrt{Q_{1}}$ yields

$$
\left|S_{1}\left(Q_{1}\right) / \mathcal{L}\right|^{2} \ll\left(N Q_{1}\right)^{2} Q_{2}^{-1}+N Q_{1} Q_{2}^{-1} \sum_{1 \leq q_{2} \leq Q_{2}}\left|S_{2}\left(q_{1}, q_{2}\right)\right|,
$$

where

$$
S_{2}\left(q_{1}, q_{2}\right):=\sum_{n \sim N} \sum_{q_{1}+q_{2}, q_{1} \sim Q_{1}} \psi_{n+q_{1}+q_{2}} \bar{\psi}_{n+q_{1}} e\left(t_{1}\left(n, q_{1}, q_{2}\right)\right)
$$

and $t_{1}\left(n, q_{1}, q_{2}\right):=\widetilde{\alpha} A^{\gamma} l^{1-\gamma}\left\{t\left(n, q_{1}+q_{2}\right)^{\gamma}-t\left(n, q_{1}\right)^{\gamma}\right\}$. Writing $n^{\prime}:=n+q_{1}$, exchanging the order of summation and using Lemma 2.6 of [12], we can deduce

$$
\begin{aligned}
S_{2}\left(q_{1}, q_{2}\right) & =\sum_{\left(n^{\prime}, q_{1}\right) \in \mathbf{D}_{2}} \sum_{n^{\prime}+q_{2}} \bar{\psi}_{n^{\prime}} e\left(t_{1}\left(n^{\prime}-q_{1}, q_{1}, q_{2}\right)\right) \\
& \ll \mathcal{L} \sum_{n^{\prime} \sim N}\left|\sum_{q_{1} \sim Q_{1}} e\left(\theta^{\prime} q_{1}\right) e\left(T\left(n^{\prime}, q_{1}, q_{2}\right)\right)\right|,
\end{aligned}
$$

where $T\left(n^{\prime}, q_{1}, q_{2}\right):=t_{1}\left(n^{\prime}-q_{1}, q_{1}, q_{2}\right), \mathbf{D}_{2}$ is a suitable subregion of $\left\{\left(n^{\prime}, q_{1}\right)\right.$ : $\left.n^{\prime} \sim N, q_{1} \sim Q_{1}\right\}$ and $\theta^{\prime}$ is a real number independent of $\left(n^{\prime}, q_{1}\right)$. A final application of Cauchy's inequality and a "Weyl shift" with $Q_{3}=Q_{2}^{2}$ gives

$$
\left|S_{2}\left(q_{1}, q_{2}\right) / \mathcal{L}\right|^{2} \ll\left(N Q_{1}\right)^{2} Q_{3}^{-1}+N Q_{1} Q_{3}^{-1} \sum_{1 \leq q_{3} \leq Q_{3}} \sum_{q_{1} \sim Q_{1}}\left|S_{3}\left(q_{1}, q_{2}, q_{3}\right)\right|,
$$

where $S_{3}\left(q_{1}, q_{2}, q_{3}\right):=\sum_{n^{\prime} \sim N} e\left(f\left(n^{\prime}\right)\right)$ and $f\left(n^{\prime}\right):=T\left(n^{\prime}, q_{1}, q_{2}\right)-T\left(n^{\prime}\right.$, $\left.q_{1}+q_{3}, q_{2}\right)$. It is easy to show that $f\left(n^{\prime}\right)$ satisfies the conditions of exponent pair and $f^{\prime}\left(n^{\prime}\right) \asymp X N^{-2} Q_{1}^{-1} q_{2} q_{3}\left(n^{\prime} \sim N\right)$. Hence we have

$$
S_{3}\left(q_{1}, q_{2}, q_{3}\right) \ll\left(X N^{-2} Q_{1}^{-1} q_{2} q_{3}\right)^{\kappa} N^{\lambda}+\left(X N^{-2} Q_{1}^{-1} q_{2} q_{3}\right)^{-1},
$$


which implies

$$
\begin{aligned}
S_{1}\left(Q_{1}\right) \ll\left\{\left(X^{\kappa} N^{3-2 \kappa+\lambda} Q_{1}^{4-\kappa} Q_{2}^{3 \kappa}\right)^{1 / 4}\right. & \\
& \left.+N Q_{1} Q_{2}^{-1 / 2}+\left(X^{-1} N^{5} Q_{1}^{5} Q_{2}^{-3}\right)^{1 / 4}\right\} \mathcal{L}^{7 / 4}
\end{aligned}
$$

provided $Q_{1} \geq \mathcal{L}, Q_{2} \leq \varepsilon_{0} \sqrt{Q_{1}}$. By Lemma 2.4(ii) of [12] optimizing $Q_{2}$ over $\left(0, \varepsilon_{0} \sqrt{Q_{1}}\right]$ yields

$$
\begin{aligned}
S_{1}\left(Q_{1}\right) \ll\left\{\left(X^{\kappa} N^{3+4 \kappa+\lambda} Q_{1}^{4+5 \kappa}\right)^{1 /(4+6 \kappa)}+\right. & N^{3 / 4+\lambda /(4+4 \kappa)} Q_{1} \\
& \left.+N Q_{1}^{3 / 4}+\left(X^{-2} N^{10} Q_{1}^{7}\right)^{1 / 8}\right\} \mathcal{L}^{7 / 4}
\end{aligned}
$$

provided $Q_{1} \geq \mathcal{L}$. In view of the term $N Q_{1}^{3 / 4} \mathcal{L}^{7 / 4}$, this inequality holds trivially when $Q_{1} \leq \mathcal{L}$. Inserting the preceding estimate in (2.4) yields, for any $Q_{1} \in[1, Q]$,

$$
\begin{aligned}
S\left(Q_{1}\right) \ll & \left\{\left(X^{2+4 \kappa} M^{-2-3 \kappa} N^{1+\kappa+\lambda} Q_{1}^{6+8 \kappa}\right)^{1 /(4+6 \kappa)}\right. \\
& +X^{1 / 2} M^{-1 / 2} N^{1 / 4+\lambda /(4+4 \kappa)} Q_{1}^{3 / 2} \\
& +\left(X^{2} M^{-2} N^{2} Q_{1}^{5}\right)^{1 / 4}+\left(X^{2} M^{-4} N^{6} Q_{1}^{11}\right)^{1 / 8}+\left(X M^{-1} N^{-1} Q_{1}^{3}\right)^{1 / 2} \\
& \left.+M^{-1 / 2} N Q_{1}+\left(X^{-1} M N Q_{1}\right)^{1 / 2}+\left(X^{-2} M N^{4}\right)^{1 / 2}\right\} \mathcal{L}^{7 / 4} \\
=: & \left(E_{1}+E_{2}+\ldots+E_{8}\right) \mathcal{L}^{7 / 4} .
\end{aligned}
$$

Since $E_{5} \leq E_{3}$ and $E_{6}=\left(E_{4}^{4} E_{8}\right)^{1 / 5}\left(M^{2} Q_{1}\right)^{-1 / 10}$, both $E_{5}$ and $E_{6}$ are superfluous. Replacing $Q_{1}$ by $Q$ and inserting the bound obtained in (2.3), we find, for any $Q \in[\mathcal{L}, N / \mathcal{L}]$,

$$
\begin{aligned}
S \ll & \left\{\left(X^{2+4 \kappa} M^{4+6 \kappa} N^{5+7 \kappa+\lambda} Q^{2+2 \kappa}\right)^{1 /(8+12 \kappa)}\right. \\
& +X^{1 / 4} M^{1 / 2} N^{5 / 8+\lambda /(8+8 \kappa)} Q^{1 / 4} \\
& +\left(X^{2} M^{4} N^{6} Q\right)^{1 / 8}+\left(X^{2} M^{8} N^{14} Q^{3}\right)^{1 / 16} \\
& \left.+M N Q^{-1 / 2}+\left(X^{-1} M^{2} N^{3} Q^{-1}\right)^{1 / 2}\right\} \mathcal{L}^{11 / 8}
\end{aligned}
$$

where we have used the fact that $\left(X^{-1} M^{4} N^{3} Q^{-1}\right)^{1 / 4}$ can be absorbed by $M N Q^{-1 / 2}$. In view of $M N Q^{-1 / 2}$, the preceding estimate holds trivially when $Q \in(0, \mathcal{L}]$.

If $X(M N)^{-1} Q_{1} \leq \varepsilon_{0}$, we can remove $m^{-1 / 2}$ by partial summation and then estimate the sum over $m$ by Kuz'min-Landau's inequality ([4], Theorem 2.1). Hence we see that $(2.5)$ always holds for $0<Q \leq N / \mathcal{L}$. Using Lemma 2.4(ii) of [12] to optimize $Q$ over $(0, N / \mathcal{L}]$ yields

$$
\begin{aligned}
S \ll & \left\{\left(X^{2+4 \kappa} M^{8+10 \kappa} N^{9+11 \kappa+\lambda}\right)^{1 /(12+16 \kappa)}\right. \\
& +\left(X^{2 \kappa} M^{8+10 \kappa} N^{11+13 \kappa+\lambda}\right)^{1 /(12+16 \kappa)} \\
& +X^{1 / 6} M^{2 / 3} N^{3 / 4+\lambda /(12+12 \kappa)} \\
& +M^{2 / 3} N^{11 / 12+\lambda /(12+12 \kappa)}+\left(X M^{3} N^{4}\right)^{1 / 5} \\
& +\left(X M^{6} N^{9}\right)^{1 / 10}+\left(X M^{7} N^{10}\right)^{1 / 11}
\end{aligned}
$$




$$
\begin{aligned}
& \left.+\left(X^{-1} M^{14} N^{23}\right)^{1 / 22}+M N^{1 / 2}+X^{-1 / 2} M N\right\} \mathcal{L}^{2} \\
= & :\left(F_{1}+F_{2}+\ldots+F_{10}\right) \mathcal{L}^{2} .
\end{aligned}
$$

Since

$$
F_{2}=\left(F_{4}^{6+3 \kappa} F_{5}^{5 \kappa}\right)^{1 /(6+8 \kappa)} N^{-\kappa(1+\kappa-\lambda) /((4+4 \kappa)(6+8 \kappa))}
$$

and $F_{6}=\left(F_{5}^{16} F_{8}^{11}\right)^{1 / 27} M^{-2 / 135}$, they are both superfluous. This proves (2.1).

To prove (2.2), we take $Q_{2}=\varepsilon_{0} \min \left\{\sqrt{Q_{1}},\left(X^{-1} N^{2} Q_{1}\right)^{1 / 3}\right\}$ such that $\left|f^{\prime}\left(n^{\prime}\right)\right| \leq 1 / 2$ for $n^{\prime} \sim N$. Thus Kuz'min-Landau's inequality gives $S_{3}\left(q_{1}, q_{2}, q_{3}\right) \ll\left(X N^{-2} Q_{1}^{-1} q_{2} q_{3}\right)^{-1}$, from which we can deduce, as before, the following inequality:

$$
\begin{aligned}
S \ll & \left\{\left(X M^{3} N^{4}\right)^{1 / 5}+\left(X M^{6} N^{9}\right)^{1 / 10}+\left(X^{4} M^{10} N^{11}\right)^{1 / 16}\right. \\
& +\left(X^{2} M^{10} N^{13}\right)^{1 / 16}+\left(X M^{7} N^{10}\right)^{1 / 11}+\left(X^{-1} M^{14} N^{23}\right)^{1 / 22} \\
& \left.+M N^{1 / 2}+X^{-1 / 2} M N+M^{1 / 2} N\right\} \mathcal{L}^{2} \\
= & :\left(G_{1}+G_{2}+\ldots+G_{9}\right) \mathcal{L}^{2} .
\end{aligned}
$$

It is not difficult to verify that $G_{2}=\left(G_{1}^{16} G_{6}^{11}\right)^{1 / 27} M^{-2 / 135}, G_{4}=$ $\left(G_{3}^{15} G_{6}^{11}\right)^{1 / 26}\left(M^{2} N^{11}\right)^{-1 / 416}, G_{9}=\left(G_{5} G_{6}^{2}\right)^{1 / 3} M^{-3 / 22}$. Thus $G_{2}, G_{4}, G_{9}$ are superfluous. This completes the proof.

For $T=T(M, N)$ defined as in Section 1, we have the following result.

Theorem 3. Let $\alpha, \beta \in \mathbb{R}$ with $\alpha \beta(\alpha-1)(\beta-1)(\alpha+\beta-1)(2 \alpha+\beta-2) \neq 0$, $X>0, M \geq 1, N \geq 1, \mathcal{L}:=\log (2+X M N),\left|\varphi_{m}\right| \leq 1$ and $I(m)$ be $a$ subinterval of $[N, 2 N]$. Then

$$
\begin{aligned}
T(M, N) \ll & \left\{\left(X^{5} M^{10} N^{8}\right)^{1 / 16}+\left(X^{3} M^{10} N^{12}\right)^{1 / 16}+\left(X M^{2} N^{3}\right)^{1 / 4}\right. \\
& +\left(X^{3} M^{14} N^{18}\right)^{1 / 22}+\left(X M^{6} N^{9}\right)^{1 / 10}+\left(X^{7} M^{30} N^{24}\right)^{1 / 40} \\
& \left.+\left(X M^{5} N^{5}\right)^{1 / 7}+M N^{1 / 2}+X^{-1} M N\right\} \mathcal{L}^{3} .
\end{aligned}
$$

Pr o of. If $X \leq \varepsilon_{0} N$, then $T \ll X^{-1} M N$ by Kuz'min-Landau's inequality. When $X \geq \varepsilon_{0} N$, using (2.3) with $\psi_{n}=1$, we have, for any $1 \leq Q \leq \varepsilon_{0} N$,

$$
|T|^{2} \ll(M N)^{2} Q^{-1}+\mathcal{L} M^{3 / 2} N Q^{-1} \max _{1 \leq Q_{1} \leq Q}\left|T\left(Q_{1}\right)\right|,
$$

where

$$
T\left(Q_{1}\right):=\sum_{q \sim Q_{1}} \sum_{n \in I_{1}(q)} \sum_{m \in J(n, q)} m^{-1 / 2} e\left(X \frac{m^{\alpha} t(n, q)}{M^{\alpha} N^{\beta}}\right),
$$

$t(n, q):=(n+q)^{\beta}-n^{\beta}, I_{1}(q)$ is a subinterval of $[N, 2 N]$ and $J(n, q)$ a subinterval of $[M, 2 M]$. 
If $L:=X(M N)^{-1} Q_{1} \geq \varepsilon_{0}$, similarly to (2.4), we can prove, for some $l \asymp L$,

$$
\begin{aligned}
T\left(Q_{1}\right) \ll & \left(X M^{-1} N^{-1} Q_{1}\right)^{1 / 2}\left|\sum_{(n, q) \in \mathbf{D}(l)} e(f(n, q))\right| \\
& +\left\{M^{-1 / 2} N Q_{1}+\left(X M^{-1} N^{-1} Q_{1}^{3}\right)^{1 / 2}\right. \\
& \left.+\left(X^{-2} M N^{4}\right)^{1 / 2}+\left(X^{-1} M N Q_{1}\right)^{1 / 2}\right\} \mathcal{L},
\end{aligned}
$$

where $f(n, q):=\widetilde{\alpha}\left(X Q_{1} / N\right)(l / L)^{\alpha /(\alpha-1)}\left\{t(n, q) /\left(N^{\beta-1} Q_{1}\right)\right\}^{1 /(1-\alpha)}$ and $\mathbf{D}(l)$ is a suitable subregion of $\left\{(n, q): n \sim N, q \sim Q_{1}\right\}$. It is easy to show that $f(n, q)$ satisfies the condition of Lemma 1.5 of $[8]$ (which is a revised form of Theorem 1 of Kolesnik [6]) with $A=X N^{-1} Q_{1} /\left(N^{\beta-1} Q_{1}\right)^{1 /(1-\alpha)}$, $\Delta=Q_{1} / N$. By this lemma with $(F, X, Y)=\left(X N^{-1} Q_{1}, N, Q_{1}\right)$, we obtain the estimate

$$
\begin{aligned}
T\left(Q_{1}\right) \ll & \left\{\left(X^{5} M^{-3} N^{-2} Q_{1}^{8}\right)^{1 / 6}+\left(X^{3} M^{-3} N^{2} Q_{1}^{8}\right)^{1 / 6}\right. \\
& +\left(X M^{-1} N Q_{1}^{2}\right)^{1 / 2}+\left(X^{3} M^{-4} N^{4} Q_{1}^{11}\right)^{1 / 8} \\
& +\left(X M^{-2} N^{3} Q_{1}^{5}\right)^{1 / 4}+\left(X^{7} M^{-5} N^{-6} Q_{1}^{20}\right)^{1 / 10} \\
& \left.+\left(X^{2} M^{-2} Q_{1}^{7}\right)^{1 / 4}+\left(X^{-2} M N^{4}\right)^{1 / 2}+\left(X^{-1} M N Q_{1}\right)^{1 / 2}\right\} \mathcal{L}^{4},
\end{aligned}
$$

where we have used the fact that $M^{-1 / 2} N Q_{1}+\left(X M^{-1} N^{-1} Q_{1}^{3}\right)^{1 / 2}$ can be absorbed by $\left(X M^{-2} N^{3} Q_{1}^{5}\right)^{1 / 4}+\left(X^{2} M^{-2} Q_{1}^{7}\right)^{1 / 4}$ (in view of the hypothesis $\left.X \geq \varepsilon_{0} N\right)$.

If $L \leq \varepsilon_{0}$, the Kuz'min-Landau inequality implies that (2.7) also holds. Replacing $Q_{1}$ by $Q$ and inserting into (2.6) yield

$$
\begin{aligned}
|T|^{2} \ll & \left\{\left(X^{5} M^{6} N^{4} Q^{2}\right)^{1 / 6}+\left(X^{3} M^{6} N^{8} Q^{2}\right)^{1 / 6}+\left(X M^{2} N^{3}\right)^{1 / 2}\right. \\
& +\left(X^{3} M^{8} N^{12} Q^{3}\right)^{1 / 8}+\left(X M^{4} N^{7} Q\right)^{1 / 4}+\left(X^{7} M^{10} N^{4} Q^{10}\right)^{1 / 10} \\
& \left.+\left(X^{2} M^{4} N^{4} Q^{3}\right)^{1 / 4}+(M N)^{2} Q^{-1}\right\} \mathcal{L}^{5},
\end{aligned}
$$

where we have eliminated two superfluous terms $X^{-1} M^{2} N^{3} Q^{-1}$ and $\left(X^{-1} M^{4} N^{3} Q^{-1}\right)^{1 / 2}$ (which can be absorbed by $\left.(M N)^{2} Q^{-1}\right)$. Using Lemma 2.4(ii) of [12] to optimize $Q$ over $\left(0, \varepsilon_{0} N\right]$ gives the required result. This concludes the proof.

Next we shall apply Theorems 2 and 3 to treat

$$
\begin{aligned}
S_{I} & :=\sum_{m_{1} \sim M_{1}} \sum_{m_{2} \sim M_{2}} \sum_{m_{3} \sim M_{3}} \psi_{m_{2}} e\left(X \frac{m_{1}^{\alpha_{1}} m_{2}^{\alpha_{2}} m_{3}^{-\alpha_{2}}}{M_{1}^{\alpha_{1}} M_{2}^{\alpha_{2}} M_{3}^{-\alpha_{2}}}\right), \\
S_{I I} & :=\sum_{m_{1} \sim M_{1}} \sum_{m_{2} \sim M_{2}} \sum_{m_{3} \sim M_{3}} \varphi_{m_{1}} \psi_{m_{2}} e\left(X \frac{m_{1}^{\alpha_{1}} m_{2}^{\alpha_{2}} m_{3}^{-\alpha_{2}}}{M_{1}^{\alpha_{1}} M_{2}^{\alpha_{2}} M_{3}^{-\alpha_{2}}}\right),
\end{aligned}
$$

which are general forms of (1.4). The following results will be used in the proof of Theorem 1. 
Corollary 1. Let $\alpha_{j} \in \mathbb{R}$ with $\alpha_{1} \alpha_{2}\left(\alpha_{2}+1\right)\left(\alpha_{1}-j \alpha_{2}-j\right) \neq 0(j=1,2)$, $X>0, M_{j} \geq 1,\left|\varphi_{m_{1}}\right| \leq 1,\left|\psi_{m_{2}}\right| \leq 1$ and let $Y:=2+X M_{1} M_{2} M_{3}$. If $(\kappa, \lambda)$ is an exponent pair, then for any $\varepsilon>0$,

$$
\begin{aligned}
S_{I I} \ll & \left\{\left(X^{4+6 \kappa} M_{1}^{9+11 \kappa+\lambda} M_{2}^{8+10 \kappa} M_{3}^{4+6 \kappa}\right)^{1 /(12+16 \kappa)}\right. \\
& +X^{1 / 3} M_{1}^{3 / 4+\lambda /(12+12 \kappa)} M_{2}^{2 / 3} M_{3}^{1 / 3}+\left(X^{3} M_{1}^{8} M_{2}^{6} M_{3}^{4}\right)^{1 / 10} \\
& +\left(X^{5} M_{1}^{20} M_{2}^{14} M_{3}^{8}\right)^{1 / 22}+X^{1 / 6} M_{1}^{11 / 12+\lambda /(12+12 \kappa)} M_{2}^{2 / 3} M_{3}^{1 / 3} \\
& +\left(X M_{1} M_{2}^{2}\right)^{1 / 2}+\left(X^{2} M_{1}^{23} M_{2}^{14} M_{3}^{8}\right)^{1 / 22}+M_{1} M_{2} \\
& \left.+X^{-1 / 2} M_{2} M_{3}+X^{-1} M_{1} M_{2} M_{3}\right\} Y^{\varepsilon} .
\end{aligned}
$$

In particular, if $X \geq M_{3} \geq M_{1}$, then

$$
\begin{aligned}
S_{I I} \ll & \left\{\left(X^{186} M_{1}^{407} M_{2}^{350} M_{3}^{186}\right)^{1 / 536}\right. \\
& +\left(X^{164} M_{1}^{385} M_{2}^{328} M_{3}^{164}\right)^{1 / 492}+\left(X^{3} M_{1}^{8} M_{2}^{6} M_{3}^{4}\right)^{1 / 10} \\
& \left.+\left(X^{5} M_{1}^{20} M_{2}^{14} M_{3}^{8}\right)^{1 / 22}+\left(X M_{1} M_{2}^{2}\right)^{1 / 2}\right\} Y^{\varepsilon} \\
S_{I I} \ll & \left\{\left(X^{13} M_{1}^{15} M_{2}^{22} M_{3}^{4}\right)^{1 / 26}+\left(X^{2} M_{1}^{2} M_{2}^{3} M_{3}\right)^{1 / 4}\right. \\
& \left.+\left(X^{9} M_{1}^{11} M_{2}^{18}\right)^{1 / 18}+\left(X M_{1}^{4} M_{2}^{3} M_{3}\right)^{1 / 4}\right\} Y^{\varepsilon} .
\end{aligned}
$$

Proof. If $M_{3}^{\prime}:=X / M_{3} \leq \varepsilon_{0}$, the Kuz'min-Landau inequality implies

$$
S_{I I} \ll X^{-1} M_{1} M_{2} M_{3} .
$$

Next we suppose $M_{3}^{\prime} \geq \varepsilon_{0}$. As before, using Lemma 2.2 of [12] to the sum over $m_{3}$ and estimating the corresponding error term by Lemma 2.3 there with $n=m_{1}$, we obtain

$S_{I I} \ll X^{-1 / 2} M_{3} S+\left(X^{1 / 2} M_{2}+M_{1} M_{2}+X^{-1 / 2} M_{2} M_{3}+X^{-1} M_{1} M_{2} M_{3}\right) \log Y$, where

$$
\begin{aligned}
S & :=\sum_{m_{1} \sim M_{1}} \sum_{m_{2} \sim M_{2}} \sum_{m_{3}^{\prime} \sim M_{3}^{\prime}} \widetilde{\varphi}_{m_{1}} \widetilde{\psi}_{m_{2}} \xi_{m_{3}^{\prime}} e\left(\widetilde{\alpha}_{2} X \frac{m_{1}^{\beta_{1}} m_{2}^{\beta_{2}} m_{3}^{\prime \beta_{2}}}{M_{1}^{\beta_{1}} M_{2}^{\beta_{2}} M_{3}^{\beta_{2}}}\right) \\
& =\sum_{m_{1} \sim M_{1}} \sum_{m_{2}^{\prime} \sim M_{2}^{\prime}} \widetilde{\varphi}_{m_{1}} \widetilde{\xi}_{m_{2}^{\prime}} e\left(\widetilde{\alpha}_{2} X \frac{m_{1}^{\beta_{1}} m_{2}^{\prime \beta_{2}}}{M_{1}^{\beta_{1}} M_{2}^{\prime \beta_{2}}}\right),
\end{aligned}
$$

and $\beta_{j}:=\alpha_{j} /\left(1+\alpha_{2}\right)(j=1,2), \widetilde{\alpha}_{2}:=\left|1+\alpha_{2}\right| \cdot\left|\alpha_{2}\right|^{-\beta_{2}},\left|\widetilde{\varphi}_{m_{1}}\right| \leq 1,\left|\widetilde{\psi}_{m_{2}}\right| \leq 1$, $\left|\xi_{m_{3}^{\prime}}\right| \leq 1, M_{2}^{\prime}:=M_{2} M_{3}^{\prime}, \widetilde{\xi}_{m_{2}^{\prime}}:=\sum \sum_{m_{2} m_{3}^{\prime}=m_{2}^{\prime}} \widetilde{\psi}_{m_{2}} \xi_{m_{3}^{\prime}}$. By Theorem 2 with $(M, N)=\left(M_{2}^{\prime}, M_{1}\right)$ we estimate $S$ to get the first assertion.

In particular taking $(\kappa, \lambda)=B A^{2}\left(\frac{1}{6}, \frac{4}{6}\right)=\left(\frac{11}{30}, \frac{16}{30}\right)$ yields

$$
\begin{aligned}
S_{I I} \ll & \left\{\left(X^{186} M_{1}^{407} M_{2}^{350} M_{3}^{186}\right)^{1 / 536}+\left(X^{164} M_{1}^{385} M_{2}^{328} M_{3}^{164}\right)^{1 / 492}\right. \\
& +\left(X^{3} M_{1}^{8} M_{2}^{6} M_{3}^{4}\right)^{1 / 10}+\left(X^{5} M_{1}^{20} M_{2}^{14} M_{3}^{8}\right)^{1 / 22} \\
& +\left(X^{82} M_{1}^{467} M_{2}^{328} M_{3}^{164}\right)^{1 / 492}+\left(X M_{1} M_{2}^{2}\right)^{1 / 2}
\end{aligned}
$$




$$
\begin{aligned}
& +\left(X^{2} M_{1}^{23} M_{2}^{14} M_{3}^{8}\right)^{1 / 22} \\
& \left.+M_{1} M_{2}+X^{-1 / 2} M_{2} M_{3}+X^{-1} M_{1} M_{2} M_{3}\right\} Y^{\varepsilon} \\
=: & \left(H_{1}+H_{2}+\ldots+H_{10}\right) Y^{\varepsilon} .
\end{aligned}
$$

Since $X \geq M_{3} \geq M_{1}$, we have $H_{5} \leq H_{2}, H_{7} \leq H_{4}, H_{j} \leq H_{6}(8 \leq j \leq 10)$ and thus $H_{5}, H_{j}(7 \leq j \leq 10)$ are superfluous. This proves (2.8).

The last inequality can be proved similarly by using Theorem 7 of [12] with $\left(M_{1}, M_{2}, M_{3}\right)=\left(M_{1}, 1, M_{2}^{\prime}\right)$. This completes the proof.

Corollary 2. Let $\alpha_{j} \in \mathbb{R}$ with $\alpha_{1} \alpha_{2}\left(\alpha_{1}-1\right)\left(\alpha_{1}-2\right)\left(\alpha_{2}+1\right)\left(\alpha_{1}-\alpha_{2}-1\right)$ $\neq 0, X>0, M_{j} \geq 1,\left|\psi_{m_{2}}\right| \leq 1$ and let $Y:=2+X M_{1} M_{2} M_{3}$. If $M_{3} \geq M_{1}$, then for any $\varepsilon>0$ we have

$$
\begin{aligned}
(2.10) S_{I} \ll & \left\{\left(X^{7} M_{1}^{8} M_{2}^{10} M_{3}^{6}\right)^{1 / 16}+\left(X^{5} M_{1}^{12} M_{2}^{10} M_{3}^{6}\right)^{1 / 16}\right. \\
& +\left(X M_{1}^{3} M_{2}^{2} M_{3}^{2}\right)^{1 / 4}+\left(X^{3} M_{1}^{9} M_{2}^{7} M_{3}^{4}\right)^{1 / 11} \\
& +\left(X^{2} M_{1}^{9} M_{2}^{6} M_{3}^{4}\right)^{1 / 10}+\left(X^{17} M_{1}^{24} M_{2}^{30} M_{3}^{10}\right)^{1 / 40} \\
& \left.+\left(X^{5} M_{1}^{10} M_{2}^{10} M_{3}^{4}\right)^{1 / 14}+\left(X M_{1} M_{2}^{2}\right)^{1 / 2}+X^{-1} M_{1} M_{2} M_{3}\right\} Y^{\varepsilon}, \\
(2.11) S_{I} \ll & \left\{\left(X^{15} M_{1}^{11} M_{2}^{22} M_{3}^{4}\right)^{1 / 26}+\left(X^{2} M_{1}^{2} M_{2}^{3} M_{3}\right)^{1 / 4}+\left(X^{3} M_{2}^{3} M_{3}\right)^{1 / 4}\right. \\
& \left.+\left(X^{11} M_{1}^{7} M_{2}^{18}\right)^{1 / 18}+M_{2} M_{3}+X^{-1} M_{1} M_{2} M_{3}\right\}(\log 2 Y)^{4} .
\end{aligned}
$$

Proof. As before we may suppose $M_{3}^{\prime}:=X / M_{3} \geq \varepsilon_{0}$ and prove

$$
\begin{aligned}
S_{I} \ll & X^{-1 / 2} M_{3} T \\
& +\left(X^{1 / 2} M_{2}+M_{1} M_{2}+X^{-1 / 2} M_{2} M_{3}+X^{-1} M_{1} M_{2} M_{3}\right) \log Y,
\end{aligned}
$$

where

$$
\begin{aligned}
T & :=\sum_{m_{1} \sim M_{1}} \sum_{m_{2} \sim M_{2}} \sum_{m_{3}^{\prime} \in I_{3}} g\left(m_{1}\right) \widetilde{\psi}_{m_{2}} \xi_{m_{3}^{\prime}} e\left(\widetilde{\alpha}_{2} X \frac{m_{1}^{\beta_{1}} m_{2}^{\beta_{2}} m_{3}^{\prime \beta_{2}}}{M_{1}^{\beta_{1}} M_{2}^{\beta_{2}} M_{3}^{\prime \beta_{2}}}\right), \\
I_{3} & :=\left[c_{3}\left(m_{1} / M_{1}\right)^{\alpha_{1}}\left(m_{2} / M_{2}\right)^{\alpha_{2}} M_{3}^{\prime}, c_{4}\left(m_{1} / M_{1}\right)^{\alpha_{1}}\left(m_{2} / M_{2}\right)^{\alpha_{2}} M_{3}^{\prime}\right],
\end{aligned}
$$

and $\beta_{j}, \widetilde{\alpha}_{2}, \widetilde{\psi}_{m_{2}}, \xi_{m_{3}^{\prime}}$ are defined as before, $c_{j}=c_{j}\left(\alpha_{2}\right)$ are constants, $g\left(m_{1}\right)$ is a monomial with $\left|g\left(m_{1}\right)\right| \leq 1$. We define $\widetilde{\xi}_{m_{2}^{\prime}}$ and $M_{2}^{\prime}$ in the same way as in the proof of Corollary 1. Exchanging the order of summation, we have

$$
T=\sum_{m_{2}^{\prime} \sim M_{2}^{\prime}} \widetilde{\xi}_{m_{2}^{\prime}} \sum_{m_{1} \in I_{1}\left(m_{2}^{\prime}\right)} g\left(m_{1}\right) e\left(\widetilde{\alpha}_{2} X \frac{m_{1}^{\beta_{1}} m_{2}^{\prime \beta_{2}}}{M_{1}^{\beta_{1}} M_{2}^{\prime \beta_{2}}}\right),
$$

where $I_{1}\left(m_{2}^{\prime}\right)$ is a subinterval of $\left[M_{1}, 2 M_{1}\right]$. Removing $g\left(m_{1}\right)$ by partial summation and estimating the double sum obtained by Theorem 3 with $(M, N)=\left(M_{2}^{\prime}, M_{1}\right)$, we find 


$$
\begin{aligned}
T \ll & \left\{\left(X^{5} M_{1}^{8} M_{2}^{\prime 10}\right)^{1 / 16}+\left(X^{3} M_{1}^{12} M_{2}^{\prime 10}\right)^{1 / 16}+\left(X M_{1}^{3} M_{2}^{\prime 2}\right)^{1 / 4}\right. \\
& +\left(X^{3} M_{1}^{18} M_{2}^{\prime 14}\right)^{1 / 22}+\left(X M_{1}^{9} M_{2}^{\prime 6}\right)^{1 / 10}+\left(X^{7} M_{1}^{22} M_{2}^{\prime 26}\right)^{1 / 36} \\
& \left.+\left(X M_{1}^{5} M_{2}^{\prime 5}\right)^{1 / 7}+M_{1}^{1 / 2} M_{2}^{\prime}\right\} Y^{\varepsilon} .
\end{aligned}
$$

Inserting into (2.12) and noticing that the last four terms on the righthand side of (2.12) can be absorbed by $\left(X M_{1} M_{2}^{2}\right)^{1 / 2}$, we obtain $(2.10)$. The inequality (2.11) is (2.7) of [13] with $\left(M_{1}, M_{2}, M_{3}\right)=\left(M_{2}, M_{1}, M_{3}\right)$ and $\left(\alpha_{1}, \alpha_{2}, \alpha_{3}\right)=\left(\alpha_{2}, \alpha_{1},-\alpha_{2}\right)$. This concludes the proof.

3. Proof of Theorem 1. We shall prove only

$$
\Delta(1,1,2 ; x) \ll_{\varepsilon} x^{47 / 131+\varepsilon},
$$

since this implies $\Delta(x) \ll_{\varepsilon} x^{47 / 131+\varepsilon}$ by a simple convolution argument. For this we recall some standard notations. Let $\mathbf{u}:=\left(u_{1}, u_{2}, u_{3}\right)$ be a permutation of $(1,1,2)$ and let $\mathbf{N}:=\left(N_{1}, N_{2}\right) \in \mathbb{N}^{2}$. We write $\psi(t):=\{t\}-1 / 2(\{t\}$ is the fractional part of $t$ ) and define

$$
S(\mathbf{u}, \mathbf{N} ; x):=\sum_{1} \psi\left(\left(x /\left(n_{1}^{u_{1}} n_{2}^{u_{2}}\right)\right)^{1 / u_{3}}\right),
$$

where the summation condition of $\sum_{1}$ is $n_{1}^{u_{1}} n_{2}^{u_{2}+u_{3}} \leq x, n_{1}(\leq) n_{2}, n_{1} \sim$ $N_{1}, n_{2} \sim N_{2}$. The notation $n_{1}(\leq) n_{2}$ means that $n_{1}=n_{2}$ for $u_{1}<u_{2}$, and $n_{1}<n_{2}$ otherwise. It is well known that for proving (3.1) it suffices to verify

$$
S(\mathbf{u}, \mathbf{N} ; x) \ll x^{47 / 131+\varepsilon} \quad \text { for } \mathbf{u}=(1,1,2),(2,1,1),(1,2,1) .
$$

Since $S(1,1,2, \mathbf{N} ; x) \ll x^{5 / 14+\varepsilon}$ (see [10], p. 263), it remains to consider $\mathbf{u}=(2,1,1),(1,2,1)$. We shall prove the desired estimate for $\mathbf{u}=(2,1,1)$ in two cases according to the size of $N_{1}$, which we shall formulate as two lemmas. The case of $\mathbf{u}=(1,2,1)$ can be treated similarly (more easily). We recall that we have $N_{1} \leq N_{2} \leq G:=x /\left(N_{1}^{2} N_{2}\right), N_{1} N_{2} \leq x^{1 / 2}$ when $\mathbf{u}=(2,1,1)$. This fact will be used (implicitly) many times in the proofs of Lemmas 3.1 and 3.2.

Lemma 3.1. For $\mathbf{u}=(2,1,1)$, we have

$$
S(\mathbf{u}, \mathbf{N} ; x) \ll_{\varepsilon}\left\{\left(x^{186} N_{1}^{35}\right)^{1 / 536}+\left(x N_{1}^{2}\right)^{1 / 4}+\left(x^{40} N_{1}^{7}\right)^{1 / 116}+x^{5 / 14}\right\} x^{\varepsilon} .
$$

In particular, if $N_{1} \leq x^{118 / 655}$, then $S(\mathbf{u}, \mathbf{N} ; x) \ll_{\varepsilon} x^{47 / 131+\varepsilon}$.

Proof. By Lemma 2.5 of [12], we have, for any $H \geq 1$,

$$
S(\mathbf{u}, \mathbf{N} ; x) \ll H^{-1} N_{1} N_{2}+(\log x) \max _{1 \leq H_{0} \leq H} H_{0}^{-1}\left|S\left(H_{0}, \mathbf{N}\right)\right|,
$$

where

$$
S\left(H_{0}, \mathbf{N}\right):=\sum_{h \sim H_{0}} a_{h} \sum_{n_{1} \sim N_{1}} \sum_{n_{2} \sim N_{2}} e\left(h x /\left(n_{1}^{2} n_{2}\right)\right), \quad\left|a_{h}\right| \leq 1 .
$$


The inequalities (2.8) and (2.9) with $\left(X, M_{1}, M_{2}, M_{3}\right)=\left(G H_{0}, N_{1}, H_{0}, N_{2}\right)$ imply

$$
\begin{aligned}
S\left(H_{0}, \mathbf{N}\right) \ll & \left\{\left(G^{186} N_{1}^{407} N_{2}^{186}\right)^{1 / 536}+\left(G N_{1} H_{0}\right)^{1 / 2}+\chi_{1}+\chi_{2}\right\} H_{0} x^{\varepsilon}, \\
S\left(H_{0}, \mathbf{N}\right) \ll & \left\{\left(G^{13} N_{1}^{15} N_{2}^{4} H_{0}^{9}\right)^{1 / 26}+\left(G^{2} N_{1}^{2} N_{2} H_{0}\right)^{1 / 4}+\left(G^{9} N_{1}^{11} H_{0}^{9}\right)^{1 / 18}\right. \\
& \left.+\left(x N_{1}^{2}\right)^{1 / 4}\right\} H_{0} x^{\varepsilon} \\
= & :\left\{D_{1}+D_{2}+D_{3}+\left(x N_{1}^{2}\right)^{1 / 4}\right\} H_{0} x^{\varepsilon},
\end{aligned}
$$

with $\chi_{1}:=\left(G^{3} N_{1}^{8} N_{2}^{4} H_{0}^{-1}\right)^{1 / 10}$ and $\chi_{2}:=\left(G^{5} N_{1}^{20} N_{2}^{8} H_{0}^{-3}\right)^{1 / 22}$, where we have used the fact that $\left(G^{164} N_{1}^{385} N_{2}^{164}\right)^{1 / 492} \leq\left(G^{186} N_{1}^{407} N_{2}^{186}\right)^{1 / 536}$ (in view of $\left.N_{1} \leq x^{1 / 4}\right)$. From these, we deduce that for any $H_{0} \geq 1$,

$$
\begin{aligned}
S\left(H_{0}, \mathbf{N}\right) \ll & \left\{\left(G^{186} N_{1}^{407} N_{2}^{186}\right)^{1 / 536}+\left(G N_{1} H_{0}\right)^{1 / 2}\right. \\
& \left.+\left(x N_{1}^{2}\right)^{1 / 4}+\sum_{1 \leq j \leq 2} \sum_{1 \leq k \leq 3} R_{j, k}\right\} H_{0} x^{\varepsilon},
\end{aligned}
$$

where $R_{j, k}:=\min \left\{\chi_{j}, D_{k}\right\}$. Since

$$
\begin{aligned}
& \left\{\begin{array}{l}
R_{1,1} \leq\left(\chi_{1}^{45} D_{1}^{13}\right)^{1 / 58}=\left(x^{40} N_{1}^{7}\right)^{1 / 116} \\
R_{1,2} \leq\left(\chi_{1}^{5} D_{2}^{2}\right)^{1 / 7}=x^{5 / 14} \\
R_{1,3} \leq\left(\chi_{1}^{5} D_{3}\right)^{1 / 6}=\left(x^{36} N_{1}^{11}\right)^{1 / 108}
\end{array}\right. \\
& \left\{\begin{array}{l}
R_{2,1} \leq\left(\chi_{2}^{33} D_{1}^{13}\right)^{1 / 46}=\left(x^{28} N_{1}^{19}\right)^{1 / 92}<x^{5 / 14} \\
R_{2,2} \leq\left(\chi_{2}^{11} D_{2}^{6}\right)^{1 / 17}=\left(x^{11} N_{1}^{4}\right)^{1 / 34}<x^{5 / 14} \\
R_{2,3} \leq\left(\chi_{2}^{11} D_{3}^{3}\right)^{1 / 14}=\left(x^{24} N_{1}^{23}\right)^{1 / 84}<x^{5 / 14}
\end{array}\right.
\end{aligned}
$$

and $\left(x^{36} N_{1}^{11}\right)^{1 / 108} \leq\left(x^{40} N_{1}^{7}\right)^{1 / 116}$, we have

$$
\begin{aligned}
S\left(H_{0}, \mathbf{N}\right) \ll & \left\{\left(G^{186} N_{1}^{407} N_{2}^{186}\right)^{1 / 536}+\left(G N_{1} H_{0}\right)^{1 / 2}\right. \\
& \left.+\left(x N_{1}^{2}\right)^{1 / 4}+\left(x^{40} N_{1}^{7}\right)^{1 / 116}+x^{5 / 14}\right\} H_{0} x^{\varepsilon} .
\end{aligned}
$$

Inserting into (3.2) and optimizing $H$ by Lemma 2.4(iii) of [12] yield the desired estimate.

Lemma 3.2. For $\mathbf{u}=(2,1,1)$, we have

$$
\begin{aligned}
S(\mathbf{u}, \mathbf{N} ; x) \ll & \left\{\left(x^{7} / N_{1}^{5}\right)^{1 / 17}+\left(x^{17} / N_{1}^{3}\right)^{1 / 47}+\left(x^{11} / N_{1}^{4}\right)^{1 / 29}\right. \\
& \left.+\left(x^{13} / N_{1}^{2}\right)^{1 / 36}+\left(x^{5} / N_{1}^{4}\right)^{1 / 12}+x^{103 / 294}\right\} x^{\varepsilon} .
\end{aligned}
$$

In particular, if $N_{1} \geq x^{118 / 655}$, then $S(\mathbf{u}, \mathbf{N} ; x) \ll_{\varepsilon} x^{47 / 131+\varepsilon}$.

Proof. Corollary 2 with $\left(X, M_{1}, M_{2}, M_{3}\right)=\left(G H_{0}, N_{1}, H_{0}, N_{2}\right)$ gives

$$
\begin{aligned}
S\left(H_{0}, \mathbf{N}\right) \ll & \left\{L\left(H_{0}\right)+\left(G^{5} N_{1}^{12} N_{2}^{6} H_{0}^{-1}\right)^{1 / 16}+\left(G N_{1}^{3} N_{2}^{2} H_{0}^{-1}\right)^{1 / 4}\right. \\
& \left.+\left(G^{3} N_{1}^{9} N_{2}^{4} H_{0}^{-1}\right)^{1 / 11}+\left(G^{2} N_{1}^{9} N_{2}^{4} H_{0}^{-2}\right)^{1 / 10}\right\} H_{0} x^{\varepsilon} \\
=: & \left\{L\left(H_{0}\right)+\sigma_{1}+\sigma_{2}+\sigma_{3}+\sigma_{4}\right\} H_{0} x^{\varepsilon},
\end{aligned}
$$




$$
\begin{aligned}
S\left(H_{0}, \mathbf{N}\right) \ll & \left\{\left(G^{15} N_{1}^{11} N_{2}^{4} H_{0}^{11}\right)^{1 / 26}+\left(G^{2} N_{1}^{2} N_{2} H_{0}\right)^{1 / 4}\right. \\
& \left.+\left(G^{3} N_{2} H_{0}^{2}\right)^{1 / 4}+\left(G^{11} N_{1}^{7} H_{0}^{11}\right)^{1 / 18}\right\} H_{0} x^{\varepsilon} \\
=: & \left\{E_{1}+E_{2}+E_{3}+\left(G^{11} N_{1}^{7} H_{0}^{11}\right)^{1 / 18}\right\} H_{0} x^{\varepsilon},
\end{aligned}
$$

where

$$
\begin{aligned}
L\left(H_{0}\right):= & \left(G^{7} N_{1}^{8} N_{2}^{6} H_{0}\right)^{1 / 16}+\left(G^{17} N_{1}^{24} N_{2}^{10} H_{0}^{7}\right)^{1 / 40} \\
& +\left(G^{5} N_{1}^{10} N_{2}^{4} H_{0}\right)^{1 / 14}+\left(G N_{1} H_{0}\right)^{1 / 2}
\end{aligned}
$$

and we have used the fact that $G^{-1} N_{1} N_{2} H_{0}^{-1}$ can be absorbed by $\left(G N_{1}^{3} N_{2}^{2} H_{0}^{-1}\right)^{1 / 4}$ in $(3.3)$, both $N_{2}$ and $G^{-1} N_{1} N_{2} H_{0}^{-1}$ by $\left(G^{3} N_{2} H_{0}^{2}\right)^{1 / 4}$ in (3.4). From (3.3) and (3.4), we deduce that for any $H_{0} \geq 1$,

$$
S\left(H_{0}, \mathbf{N}\right) \ll\left\{L\left(H_{0}\right)+\left(G^{11} N_{1}^{7} H_{0}^{11}\right)^{1 / 18}+\sum_{1 \leq j \leq 4} \sum_{1 \leq k \leq 3} S_{j, k}\right\} H_{0} x^{\varepsilon},
$$

where $S_{j, k}:=\min \left\{\sigma_{j}, E_{k}\right\}$. It is easy to verify that

$$
\begin{aligned}
& \left\{\begin{array}{l}
S_{1,1} \leq\left(\sigma_{1}^{88} E_{1}^{13}\right)^{1 / 101}=\left(x^{70} N_{1}^{3}\right)^{1 / 202}<x^{103 / 294}, \\
S_{1,2} \leq\left(\sigma_{1}^{4} E_{2}\right)^{1 / 5}=x^{7 / 20}<x^{103 / 294}, \\
S_{1,3} \leq\left(\sigma_{1}^{8} E_{3}\right)^{1 / 9}=\left(x^{13} / N_{1}^{2}\right)^{1 / 36},
\end{array}\right. \\
& \left\{\begin{array}{l}
S_{2,1} \leq\left(\sigma_{2}^{22} E_{1}^{13}\right)^{1 / 35}=\left(x^{13} / N_{1}^{4}\right)^{1 / 35}, \\
S_{2,2} \leq\left(\sigma_{2} E_{2}\right)^{1 / 2}=\left(x^{3} / N_{1}\right)^{1 / 8}, \\
S_{2,3} \leq\left(\sigma_{2}^{2} E_{3}\right)^{1 / 3}=\left(x^{5} / N_{1}^{4}\right)^{1 / 12},
\end{array}\right. \\
& \left\{\begin{array}{l}
S_{3,1} \leq\left(\sigma_{3}^{121} E_{1}^{26}\right)^{1 / 147}=\left(x^{48} N_{1}^{14}\right)^{1 / 147} \leq x^{103 / 294}, \\
S_{3,2} \leq\left(\sigma_{3}^{11} E_{2}^{4}\right)^{1 / 15}=\left(x^{5} N_{1}\right)^{1 / 15}<x^{103 / 294}, \\
S_{3,3} \leq\left(\sigma_{3}^{11} E_{3}^{2}\right)^{1 / 13}=x^{9 / 26}<x^{103 / 294},
\end{array}\right. \\
& \left\{\begin{array}{l}
S_{4,1} \leq\left(\sigma_{4}^{55} E_{1}^{26}\right)^{1 / 81}=\left(x^{52} N_{1}^{17}\right)^{1 / 162}<x^{103 / 294}, \\
S_{4,2} \leq\left(\sigma_{4}^{5} E_{2}^{4}\right)^{1 / 9}=\left(x^{6} N_{1}\right)^{1 / 18}<x^{103 / 294}, \\
S_{4,3} \leq\left(\sigma_{4}^{5} E_{3}^{2}\right)^{1 / 7}=\left(x^{5} / N_{1}\right)^{1 / 14},
\end{array}\right.
\end{aligned}
$$

and $\left(x^{13} / N_{1}^{4}\right)^{1 / 35} \leq\left(x^{3} / N_{1}\right)^{1 / 8},\left(x^{5} / N_{1}\right)^{1 / 14} \leq\left(x^{3} / N_{1}\right)^{1 / 8}$. Consequently, we obtain, for any $H_{0} \geq 1$, the inequality

$$
\begin{aligned}
S\left(H_{0}, \mathbf{N}\right) \ll & \left\{L\left(H_{0}\right)+\left(G^{11} N_{1}^{7} H_{0}^{11}\right)^{1 / 18}+\left(x^{13} / N_{1}^{2}\right)^{1 / 36}\right. \\
& \left.+\left(x^{3} / N_{1}\right)^{1 / 8}+\left(x^{5} / N_{1}^{4}\right)^{1 / 12}+x^{103 / 294}\right\} H_{0} x^{\varepsilon} .
\end{aligned}
$$

Inserting this estimate in (3.2) and using Lemma 2.4(iii) of [12] to optimize $H$, we find

$$
\begin{aligned}
S(\mathbf{u}, \mathbf{N} ; x) \ll & \left\{\left(x^{7} / N_{1}^{5}\right)^{1 / 17}+\left(x^{17} / N_{1}^{3}\right)^{1 / 47}+\left(x^{11} / N_{1}^{4}\right)^{1 / 29}\right. \\
& \left.+\left(x^{13} / N_{1}^{2}\right)^{1 / 36}+\left(x^{3} / N_{1}\right)^{1 / 8}+\left(x^{5} / N_{1}^{4}\right)^{1 / 12}+x^{103 / 294}\right\} x^{\varepsilon} .
\end{aligned}
$$

Observing that $\left(x^{3} / N_{1}\right)^{1 / 8} \leq\left(x^{11} / N_{1}^{4}\right)^{1 / 29}$, we get the required estimate. 


\section{References}

[1] R. C. Baker and G. Harman, Numbers with a large prime factor, Acta Arith. 73 (1995), 119-145.

[2] E. Cohen, On the average number of direct factors of a finite abelian group, ibid. 6 (1960), 159-173.

[3] E. Fouvry and H. Iwaniec, Exponential sums with monomials, J. Number Theory 33 (1989), 311-333.

[4] S. W. Graham and G. Kolesnik, Van der Corput's Method of Exponential Sums, Cambridge Univ. Press, Cambridge, 1991.

[5] C. H. Jia, The distribution of square-free numbers, Sci. China Ser. A 36 (1993), 154-169.

[6] G. Kolesnik, On the number of abelian groups of a given order, J. Reine Angew. Math. 329 (1981), 164-175.

[7] E. Krätzel, On the average number of direct factors of a finite abelian group, Acta Arith. 51 (1988), 369-379.

[8] H.-Q. Liu, The greatest prime factor of the integers in an interval, ibid. 65 (1993), 302-328.

[9] —, On some divisor problems, ibid. 68 (1994), 193-200.

[10] - Divisor problems of 4 and 3 dimensions, ibid. 73 (1995), 249-269.

[11] P. G. Schmidt, Zur Anzahl unitärer Faktoren abelscher Gruppen, ibid. 64 (1993), 237-248.

[12] P. Sargos and J. Wu, Multiple exponential sums with monomials and their applications in number theory, Prépublications $97 / n^{\circ} 37$ de l'Institut Élie Cartan, Université Henri Poincaré (Nancy 1).

[13] J. Wu, On the distribution of square-full and cube-full integers, Monatsh. Math., to appear.

Laboratoire de Mathématiques

Institut Élie Cartan - CNRS UMR 9973

Université Henri Poincaré (Nancy 1)

54506 Vandœuvre-lès-Nancy, France

E-mail: wujie@iecn.u-nancy.fr 